\title{
EXPANDED VERY LARGE ARRAY OBSERVATIONS OF A PROTO-CLUSTER OF MOLECULAR GAS-RICH GALAXIES AT $z=4.05^{*}$
}

\author{
C. L. Carilli ${ }^{1}$, J. Hodge ${ }^{2}$, F. Walter ${ }^{2}$, D. Riechers ${ }^{3,7}$, E. Daddi ${ }^{4}$, H. Dannerbauer ${ }^{2}$, And G. E. Morrison ${ }^{5,6}$ \\ ${ }^{1}$ National Radio Astronomy Observatory, P.O. Box 0, Socorro, NM 87801-0387, USA \\ ${ }^{2}$ Max-Planck Institute for Astronomy, Konigstuhl 17, 69117, Heidelberg, Germany \\ ${ }^{3}$ Department of Astronomy, California Institute of Technology, MC 249-17, 1200 East California Boulevard, Pasadena, CA 91125, USA \\ ${ }^{4}$ Laboratoire AIM, CEA/DSM-CNRS-University Paris Diderot, DAPNIA/Service Astrophysccique, CEA Saclay, \\ Orme des Merisiers, 91191 Gif-sur-Yvette, France \\ ${ }^{5}$ Institute for Astronomy, University of Hawaii, Honolulu, HI 96822, USA \\ ${ }^{6}$ Canada-France-Hawaii Telescope, Kamuela, HI 96743, USA \\ Received 2011 April 15; accepted 2011 May 17; published 2011 August 29
}

\begin{abstract}
We present observations of the molecular gas in the GN20 proto-cluster of galaxies at $z=4.05$ using the Expanded Very Large Array (EVLA). This group of galaxies is the ideal laboratory for studying the formation of massive galaxies via luminous, gas-rich starbursts within $1.6 \mathrm{Gyr}$ of the big bang. We detect three galaxies in the protocluster in $\mathrm{CO} 2-1$ emission, with gas masses $\left(\mathrm{H}_{2}\right)$ between $10^{10}$ and $10^{11} \times(\alpha / 0.8) M_{\odot}$. The emission from the brightest source, GN20, is resolved with a size $\sim 2^{\prime \prime}$ and has a clear north-south velocity gradient, possibly indicating ordered rotation. The gas mass in GN20 is comparable to the stellar mass $\left(1.3 \times 10^{11} \times(\alpha / 0.8) M_{\odot}\right.$ and $2.3 \times 10^{11} M_{\odot}$, respectively), and the sum of gas plus stellar mass is comparable to the dynamical mass of the system $\left(\sim 3.4 \times 10^{11}\left[\sin (i) / \sin \left(45^{\circ}\right)\right]^{-2} M_{\odot}\right)$, within a $5 \mathrm{kpc}$ radius. There is also evidence for a tidal tail extending another $2^{\prime \prime}$ north of the galaxy with a narrow velocity dispersion. GN20 may be a massive, gas-rich disk that is gravitationally disturbed, but not completely disrupted. There is one Lyman-break galaxy (BD29079) in the GN20 proto-cluster with an optical spectroscopic redshift within our search volume, and we set a $3 \sigma$ limit to the molecular gas mass of this galaxy of $1.1 \times 10^{10} \times(\alpha / 0.8) M_{\odot}$.
\end{abstract}

Key words: galaxies: evolution - galaxies: ISM - galaxies: starburst - submillimeter: galaxies

Online-only material: color figure

\section{INTRODUCTION}

Numerous lines of evidence support the hypothesis that massive elliptical galaxies form the majority of their stars quickly at early epochs, including stellar population synthesis studies of nearby elliptical galaxies (Renzini 2006; Collins et al. 2009), a faster decline with cosmic time in the star formation rate per unit stellar mass with increasing galaxy mass (Moresco et al. 2010), and the direct observation of "red and dead" ellipticals in clusters at high redshift (Kurk 2009; Doherty et al. 2009; Andreon \& Huertas-Company 2011). Plausible progenitors for these evolved galaxies at high redshift are even higher redshift submillimeter galaxies (SMGs), corresponding to dusty, luminous starburst galaxies found in wide-field submillimeter surveys (Blain et al. 2002). These hyper-luminous high- $z$ galaxies may trace high overdensities and are likely related to the formation of clusters and large ellipticals (Stevens et al. 2003; Aravena et al. 2010; although cf. Chapman et al. 2009; Williams et al. 2011). While the space density of SMGs peaks around $z \sim 2.3$ (Chapman et al. 2003), it is becoming clear that there is a substantial high-redshift tail of the SMG population, extending to $z>4$ (Capak et al. 2011; Riechers et al. 2010; Wardlow et al. 2011). Daddi et al. (2009b) conclude, based on SMG space densities and duty cycles, that there are likely enough SMGs at $z>3.5$ to account for the known populations of old massive galaxies at $z \sim 2-3$.

A key question for the SMGs is: what drives the prolific star formation? Tacconi et al. $(2006,2008)$ argue, based on imaging

\footnotetext{
* The Very Large Array of the National Radio Astronomy Observatory is a facility of the National Science Foundation operated under cooperative agreement by Associated Universities, Inc.

7 Hubble Fellow.
}

of higher order $\mathrm{CO}$ emission from a sample of $z \sim 2 \mathrm{SMGs}$, that SMGs are predominantly nuclear starbursts, with median sizes $<0$ '.5 ( $<4 \mathrm{kpc}$ ), "representing extreme, short-lived, maximum star-forming events in highly dissipative mergers of gas-rich galaxies." This conclusion is supported by very long baseline interferometry imaging of the star-forming regions in two SMGs (Momjian et al. 2005, 2010; Biggs et al. 2010). However, recent imaging of the lower order CO emission in a few SMGs (Ivison et al. 2010, 2011; Carilli et al. 2010; Riechers et al. 2011) suggests that the lower-excitation molecular gas reservoirs can be significantly more extended.

Daddi et al. (2009b) have identified a unique region in GOODS-N, containing the three galaxies: GN20, GN20.2a, and GN20.2b, at $z \sim 4.05 \pm 0.01$, within $20^{\prime \prime}$ of each other (projected physical separation $=140 \mathrm{kpc}$ ). GN20 is among the brightest SMGs known, with a $350 \mathrm{GHz}$ flux density of $23 \mathrm{mJy}$. The galaxies GN20.2a and b are separated by only a few arcseconds, and hence are not spatially distinct in typical submillimeter bolometer images. The two galaxies have a summed flux density of $S_{350 \mathrm{GHz}} \sim 9.9 \mathrm{mJy}$. The implied IR luminosities $(8-1000 \mu \mathrm{m})$ are $L_{\mathrm{TIR}} \geqslant 10^{13} L_{\odot}$, with star formation rates $>1000 M_{\odot} \mathrm{yr}^{-1}$ (Daddi et al. 2009b). Highorder $\mathrm{CO}$ emission (4-3 and/or 6-5) has been detected from all three galaxies, although only marginally in the case of GN20.2b (Daddi et al. 2009b).

The GN20 field also contains numerous Lyman-break galaxies (LBGs) at similar redshift and an overdensity of $z_{\text {phot }}>3.5$ Infrared Array Camera selected galaxies (Daddi et al. 2009b). Hence, this field offers an unprecedented opportunity to perform a definitive study of the gas distribution, kinematics, and physical conditions in a proto-cluster of galaxies within $1.6 \mathrm{Gyr}$ of the big bang. In this Letter, we present a survey with the 
Expanded Very Large Array (EVLA; Perley et al. 2011) of the CO 2-1 emission from the GN20 proto-cluster. The low order transitions are critical for determining the total gas mass. We also search for $\mathrm{CO}$ emission from other galaxies in the cosmic volume surveyed.

\section{THE GN20 PROTO-CLUSTER: A MASSIVE GALAXY FORMATION LABORATORY}

The Hubble Space Telescope (HST) I-band image of the bright SMG GN20 shows diffuse emission about 1".5 in extent (Daddi et al. 2009b), although offset from the radio and submillimeter emission by $\sim 1^{\prime \prime}$, implying high obscuration of the most active star-forming regions in the galaxy. GN20 is detected at $1.4 \mathrm{GHz}$ and resolved on a scale of $\sim 1^{\prime \prime} .5$, with a total flux density at $72 \pm 13 \mu \mathrm{Jy}$ (Morrison et al. 2010; Casey et al. 2009). Highresolution imaging of the $850 \mu \mathrm{m}$ emission shows resolved structure, with a north-south extension possibly as large as 1".5 (Younger et al. 2008; Iono et al. 2006). The $6.2 \mu \mathrm{m}$ polycyclic aromatic hydrocarbon spectral feature has been detected in GN20 using Spitzer (D. Riechers et al. 2011, in preparation). The integrated CO 1-0 and 2-1 emission from GN20 has been imaged at resolutions down to 0'.2 with the Very Large Array (VLA; Carilli et al. 2010). The molecular gas is extended on a scale of at least 1".5, and at high resolution forms a partial ring, or disk. Unfortunately, the old VLA correlator provided no velocity information. The excitation of the integrated $\mathrm{CO}$ emission from GN20 is higher than the Milky Way, but lower than high-redshift quasar hosts and the nuclear starburst regions of IR-luminous galaxies. The CO 4-3 line strength in GN20 is more than a factor of two lower than expected for thermal excitation (Carilli et al. 2010).

GN20.2a shows a complex and extended morphology in the HST images (Daddi et al. 2009b), and it is a relatively strong radio source $\left(S_{1.4}=181 \mu \mathrm{Jy}\right)$, likely corresponding to a radio AGN. The spectral energy distribution (SED) in the optical through near-IR is consistent with a star-forming galaxy. GN20.2a has been detected in CO 4-3 emission by Daddi et al. (2009b). Daddi et al. derive an IR luminosity $(8-1000 \mu \mathrm{m})$ of $L_{\mathrm{IR}}=1.6 \times 10^{13} L_{\odot}$.

GN20.2b is faint and compact in the HST image. It is also detected in the radio continuum at $S_{1.4}=32 \mu \mathrm{Jy}$, consistent with star formation. $\mathrm{CO} 4-3$ emission is marginally detected with the Plateau de Bure Interferometer (PdBI) and appears to be broad (700 $\mathrm{km} \mathrm{s}^{-1}$; Daddi et al. 2009b).

Daddi et al. (2009b) find $15 B$-band dropout galaxies in a $25^{\prime \prime}$ radius centered on GN20, an overdensity of a factor of six compared with the full GOODS-N area, which is significant at the $7 \sigma$ level. A spike in the redshift distribution of galaxies at $z=4.06 \pm 0.02$ is observed in all of GOODS-N (13 spectroscopic redshifts in total at this redshift). Lastly, the SMG GN10 at $z=4.04$ is located 9' from GN20 (Daddi et al. 2009a; Dannerbauer et al. 2008). Therefore, it appears that the GN20 volume has a very significant overdensity, indicating a protocluster environment at $z \sim 4.05$. Daddi et al. (2009b) estimate a total mass for this structure of $\sim 10^{14} M_{\odot}$.

\section{OBSERVATIONS}

We observed the GN20 field with the EVLA in the D $(1 \mathrm{~km})$ configuration in 2010 March and April. Observations were made of the CO 2-1 line, using a total bandwidth of $246 \mathrm{MHz}$ and in each of two polarizations, centered at $45.655 \mathrm{GHz}$. The velocity coverage is $1600 \mathrm{~km} \mathrm{~s}^{-1}$, including the $\mathrm{CO} 2-1$ lines in the three galaxies. The total observing time was $28 \mathrm{hr}$, but only about 22 to 24 antennas were available at a time due to ongoing EVLA work.

Dynamic scheduling to ensure good weather is now standard at the EVLA. Fast switching phase calibration was employed (Carilli \& Holdaway 1999) on timescales between two and three minutes using the VLA calibrator J1302+5748. Data were edited to remove time ranges of poor phase stability. The source 3C286 was used for flux density calibration. Standard EVLA data calibration and editing were performed using AIPS. After calibration and data editing, images were synthesized using the robust weighting scheme of the $u v$ data with $R=2$ (Cornwell et al. 1999).

The final resolution for the images was $1^{\prime \prime} .9$. Spectral line cubes were generated at $12 \mathrm{MHz}$ channel $^{-1}\left(78 \mathrm{~km} \mathrm{~s}^{-1}\right)$. The rms noise per channel at $12 \mathrm{MHz}$ resolution is $0.11 \mathrm{mJy}^{-1}$ beam ${ }^{-1}$. All images were corrected for the VLA primary beam response, which has a FWHM $\sim 1^{\prime}$ at $45 \mathrm{GHz}$. The field pointing center was located $10^{\prime \prime}$ west of GN20.

\section{RESULTS}

Figure 1 shows the CO 2-1 emission integrated over the full frequency range covered by these observations for the GN20 group. The crosses mark the $1.4 \mathrm{GHz}$ continuum positions of the three galaxies GN20, 20.2a,b (Morrison et al. 2010), and the optical position of one LBG with a spectroscopic redshift in the redshift range of the CO 2-1 observations (Section 5.2). We have added the previously published D array observations (Carilli et al. 2010) to improve the signal-to-noise ratio $(\mathrm{S} / \mathrm{N})$. Unfortunately, the previous data did not have the exact same velocity coverage, and hence the velocity-integrated flux densities are not accurate. Hence, this image acts as a finding chart for the CO-emitting regions, but accurate line fluxes will be based on subsequent analysis of the new EVLA data itself.

We detect CO 2-1 emission from GN20, GN20.2a, and GN20.2b. GN20 is extended on a scale $\sim 2^{\prime \prime}$ (see below). GN20.2a is not spatially resolved by these observations. GN20.2b may be extended, although the $\mathrm{S} / \mathrm{N}$ of these data is not conclusive.

Figure 2 shows spectra of the three sources. For GN20, we integrate over an area of $2^{\prime \prime}$. For the other two sources, we only consider the spectra at the peak of the velocity-integrated $\mathrm{CO}$ image. Results for Gaussian fits to the lines are given in Table 1. The lines are broad for GN20 and GN20.2a $\left(\sim 800 \mathrm{~km} \mathrm{~s}^{-1}\right)$, although not outside the range of the zero intensity line widths seen in some nearby ULIRGs (Downes \& Solomon 1998). However, we note that due to the bandwidth limitation for the early EVLA the very lowest frequency (highest velocity) emission for GN20 may be truncated by these observations, although the higher order $\mathrm{CO}$ transitions show that we expect little emission beyond our lowest spectral channel (Daddi et al. 2009b). GN20.2b shows a narrower profile at the peak position. However, the detection in the velocity-integrated image over the full frequency range (Figure 1) suggests that the emission may be extended both spatially and spectrally. A possibly broader line is also suggested for the higher order $\mathrm{CO}$ emission, but again, the high-order CO detection is only marginal (Daddi et al. 2009b). We have investigated this possibility with the current data, and conclude that more sensitive observations are required to better characterize the $\mathrm{CO}$ emission from GN20.2b, and we simply present the spectrum at the peak position herein.

Figure 3 shows the velocity channel images for the GN20 CO 2-1 emission at $78 \mathrm{~km} \mathrm{~s}^{-1}$ channel $^{-1}$ and $1^{\prime \prime} .9$ resolution. 


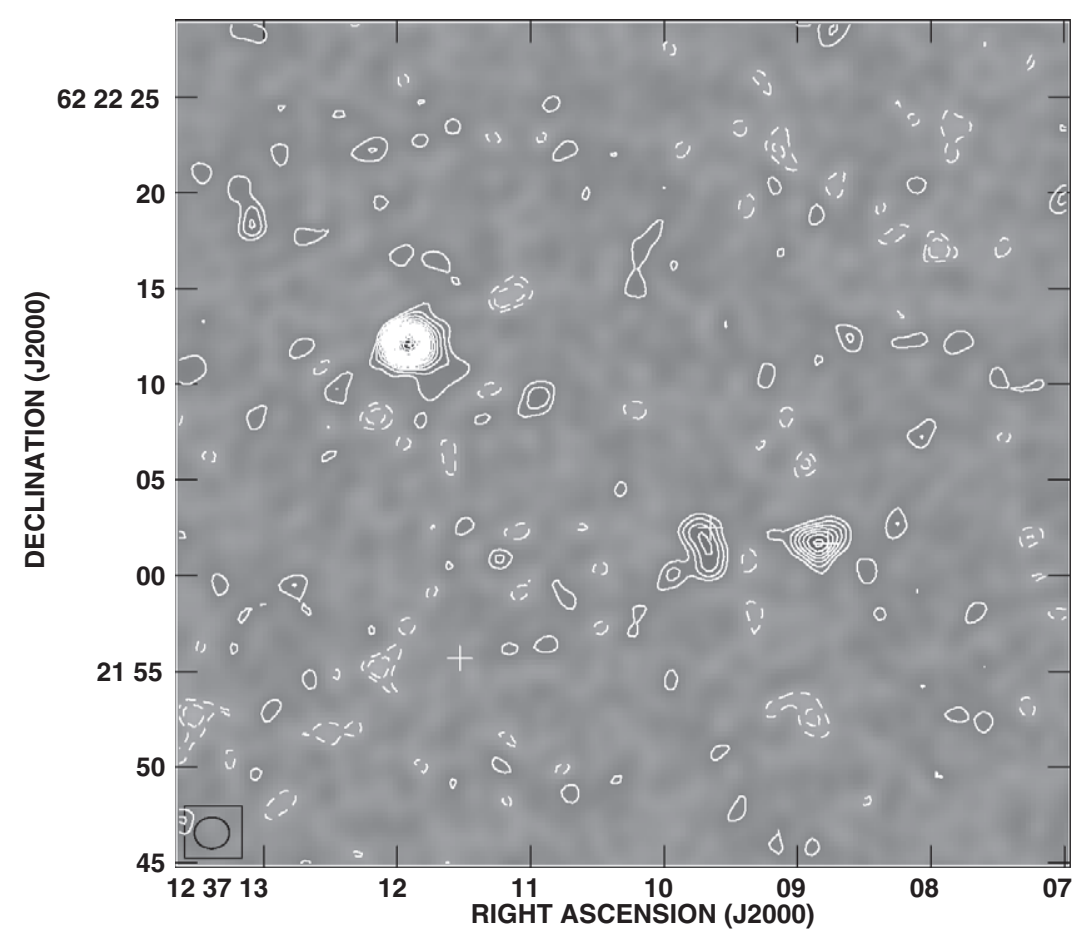

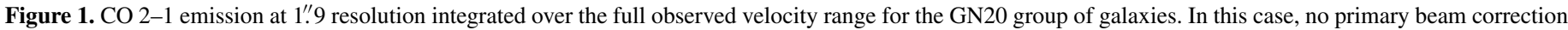

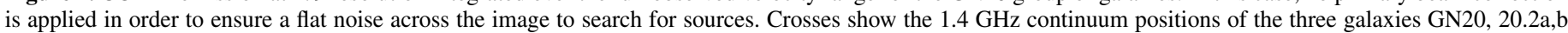

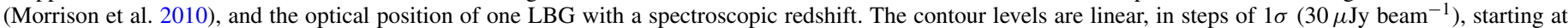
$\pm 2 \sigma$. Negative contours are dashed.

Table 1

Results from Gaussian Fitting to the CO 2-1 Line Profiles

\begin{tabular}{|c|c|c|c|c|c|c|c|}
\hline Source & Redshift & $\begin{array}{l}\text { Peak } \\
(\mathrm{mJy})\end{array}$ & $\begin{array}{l}\text { FWHM } \\
\left(\mathrm{km} \mathrm{s}^{-1}\right)\end{array}$ & $\begin{array}{c}I_{\mathrm{CO}} \\
\left(\mathrm{Jy} \mathrm{km} \mathrm{s}^{-1}\right)\end{array}$ & $\begin{array}{l}\text { Velocity }^{\mathrm{a}} \\
\left(\mathrm{km} \mathrm{s}^{-1}\right)\end{array}$ & $\begin{array}{c}L_{\mathrm{CO}}^{\prime} \\
\left(\mathrm{K} \mathrm{km} \mathrm{s}^{-1} \mathrm{pc}^{2}\right)\end{array}$ & $\begin{array}{c}M\left(\mathrm{H}_{2}\right) \\
\left(M_{\odot}\right)\end{array}$ \\
\hline GN20 & 4.0554 & $1.21 \pm 0.11$ & $679 \pm 88$ & $0.87 \pm 0.088$ & $-11 \pm 32$ & $1.6 \times 10^{11}$ & $1.3 \times 10^{11}$ \\
\hline GN20.2a & 4.0508 & $0.54 \pm 0.065$ & $723 \pm 110$ & $0.41 \pm 0.050$ & $-283 \pm 45$ & $7.6 \times 10^{10}$ & $6.1 \times 10^{10}$ \\
\hline GN20.2b & 4.0567 & $0.42 \pm 0.083$ & $290 \pm 70$ & $0.13 \pm 0.025$ & $67 \pm 29$ & $2.4 \times 10^{10}$ & $1.9 \times 10^{10}$ \\
\hline
\end{tabular}

Note. ${ }^{\text {a }}$ Velocity relative to $z=4.0556$.

The emission clearly moves from the south to the north with an increasing frequency. An interesting feature is seen in channel 6, corresponding to a frequency of $45.598 \mathrm{GHz}$ (or $z=4.05588$ ). The emission in channel 6 appears to be substantially more extended than in other channels. Inspecting our bandpass calibration, we find no channel-dependent calibration errors that would lead to such a difference in morphology for this single channel. In channel 6 the emission extends well to the north, with a total of extent of $\sim 4^{\prime \prime}$. Admittedly, the $\mathrm{S} / \mathrm{N}$ is not high, but this extended emission is suggestive of a gravitationally induced tidal feature. Such tidal tails in nearby galaxies often occur over a narrow velocity range (Hibbard \& Mihos 1995).

Figure 4(a) shows the CO 2-1 emission from GN20 integrated over the velocity range shown in the channel maps of Figure 3. The emission appears extended, in particular to the southwest. A formal Gaussian fit to the emission yields a total flux density of $0.69 \pm .07 \mathrm{mJy}$, a peak surface brightness of $0.49 \pm 0.03$ $\mathrm{Jy} \mathrm{beam}^{-1}$, and a deconvolved source size of $1^{\prime \prime} .5 \times 0.9$ with major axis position angle (P.A.) $=69^{\circ}$. Figure $4(\mathrm{~b})$ shows the iso-velocity contours (first moment) of the CO 2-1 emission, i.e., the weighted mean velocity derived after first blanking the channel images below $3 \sigma$ channel $^{-1}$. There is a velocity gradient north-south, with a magnitude of $\pm 250 \mathrm{~km} \mathrm{~s}^{-1}$. Given the low spatial resolution of these images, this corresponds to a lower limit to the true projected rotational velocity.

\section{ANALYSIS}

\subsection{Masses}

We derive the mass in molecular gas $\left(\mathrm{H}_{2}\right)$ from the observed CO 2-1 luminosities using the standard relationships in Solomon \& Vanden Bout (2005). We extrapolate from CO 2-1 to 1-0 luminosity assuming constant brightness temperature, which is certainly correct to within $10 \%$ for these galaxies (Carilli et al. 2010). We adopt a $\mathrm{CO}$ luminosity to $\mathrm{H}_{2}$ conversion factor of $\alpha=0.8 M_{\odot} \mathrm{K} \mathrm{km} \mathrm{s}^{-1} \mathrm{pc}^{2}$, appropriate for nearby nuclear starburst galaxies (Downes \& Solomon 1998). The resulting values are listed in Table 1.

Admittedly, the uncertainty on $\alpha$ is significant. A minimum gas mass can be derived assuming optically thin emission, and adopting a temperature and a $\mathrm{CO}$ to $\mathrm{H}_{2}$ abundance ratio. Ivison et al. (2011) show that for reasonable assumptions (Galactic CO abundance in molecular clouds, and temperatures $40-50 \mathrm{~K}$ ), the lower limit to $\alpha \sim 0.65$ (see also Aalto et al. 1995).

The gas mass in GN20 is $1.3 \times 10^{11} \times(\alpha / 0.8) M_{\odot}$. Daddi et al. (2009b) derive a stellar mass of $2.3 \times 10^{11} M_{\odot}$ from IR through optical SED fitting.

We can obtain a very rough estimate of the gravitational mass of GN20 from the CO velocity field, although there are substantial uncertainties due to the low spatial resolution of these 


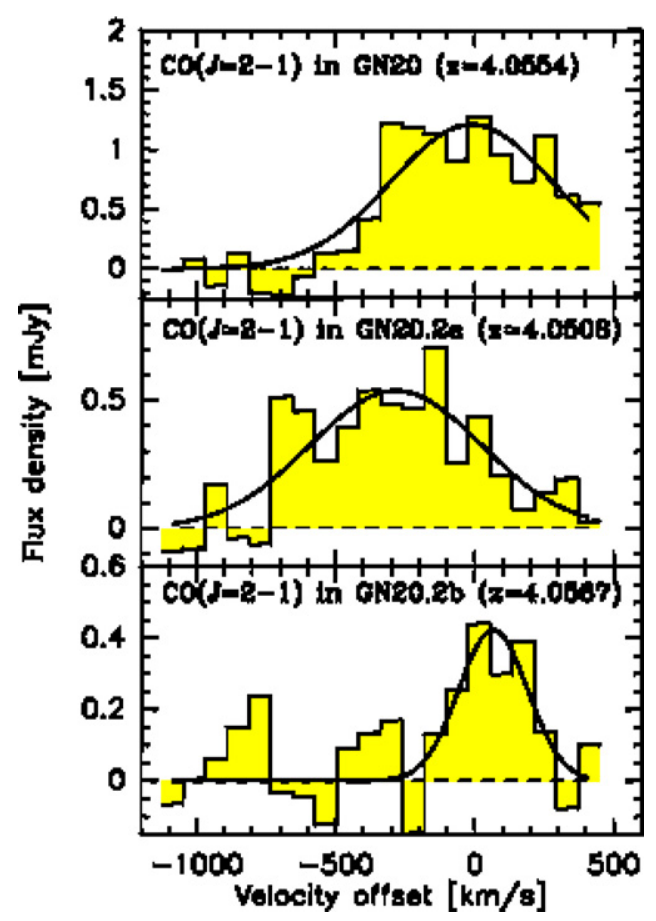

Figure 2. CO 2-1 spectra for GN20, GN20.2a, and GN20.2b at $78 \mathrm{~km} \mathrm{~s}^{-1}$ spectral resolution. Results for the Gaussian fitting are given in Table 1.

(A color version of this figure is available in the online journal.)

observations. For the rotational velocity, we adopt the extremes of the velocity channels with significant emission in Figure 3, or $390 / \sin (i) \mathrm{km} \mathrm{s}^{-1}$. For the radius, we adopt the value for the $\mathrm{CO}$ ring seen in the high-resolution 2-1 observations presented in Carilli et al. (2010), $\sim 5 \mathrm{kpc}$. The inclination angle remains uncertain. The gravitational mass inside this radius is $\sim 3.4 \times 10^{11}\left[\sin (i) / \sin \left(45^{\circ}\right)\right]^{-2} M_{\odot}$. This naively derived dynamical mass is comparable to the sum of the stellar and gas masses.

\subsection{Lyman-break Galaxy Molecular Mass Limits}

There is one LBG within our field with a spectroscopic redshift placing the $\mathrm{CO} 2-1$ line within our band: $\mathrm{J} 123711.48+622155.8$ at $z=4.058$ (BD29079; Daddi et al. 2009b; Shim et al. 2011). We find no CO emission from this galaxy, to a $1 \sigma$ limit of $0.17 \mathrm{mJy}_{\text {beam }}^{-1}$ at $78 \mathrm{~km} \mathrm{~s}^{-1}$ channel $^{-1}$, after primary beam correction. Convolving to $312 \mathrm{~km} \mathrm{~s}^{-1}$, we set a $3 \sigma$ limit to the $\mathrm{CO} 1-0$ luminosity of $L_{\mathrm{CO} 1-0}^{\prime}<$ $1.4 \times 10^{10} \mathrm{~K} \mathrm{~km} \mathrm{~s}^{-1} \mathrm{pc}^{2}$, assuming constant brightness temperature from $\mathrm{CO} 2-1$ to $1-0$. The implied molecular gas mass of this galaxy is $<1.1 \times 10^{10} \times(\alpha / 0.8) M_{\odot}$. The stellar mass of this galaxy is $2.6 \times 10^{10} M_{\odot}$ (Daddi et al. 2009b), hence the gas-to-stellar mass ratio is $<0.53 \times(\alpha / 0.8)$.

Daddi et al. (2009b) estimate a star formation rate of $150 M_{\odot}$ year $^{-1}$ for BD29079, or an IR luminosity of $1.5 \times$ $10^{12} L_{\odot}$. Daddi et al. (2010) have considered the "star formation law" in high- $z$ galaxies, i.e., the relationship between IR luminosity and $\mathrm{CO}$ luminosity, and find for galaxies of this luminosity at $z \sim 2: L_{\mathrm{CO} 1-0}^{\prime} \sim 0.02 L_{\mathrm{FIR}} \mathrm{K} \mathrm{km} \mathrm{s}^{-1} \mathrm{pc}^{2}$. Adopting this relation implies an expected $\mathrm{CO}$ luminosity of $L_{\mathrm{CO} 1-0}^{\prime} \sim 3 \times 10^{10} L_{\odot}$. Hence, BD29079 is underluminous in $\mathrm{CO}$ emission relative to the standard star formation law, although within the broad (factor three) scatter at any given luminosity.

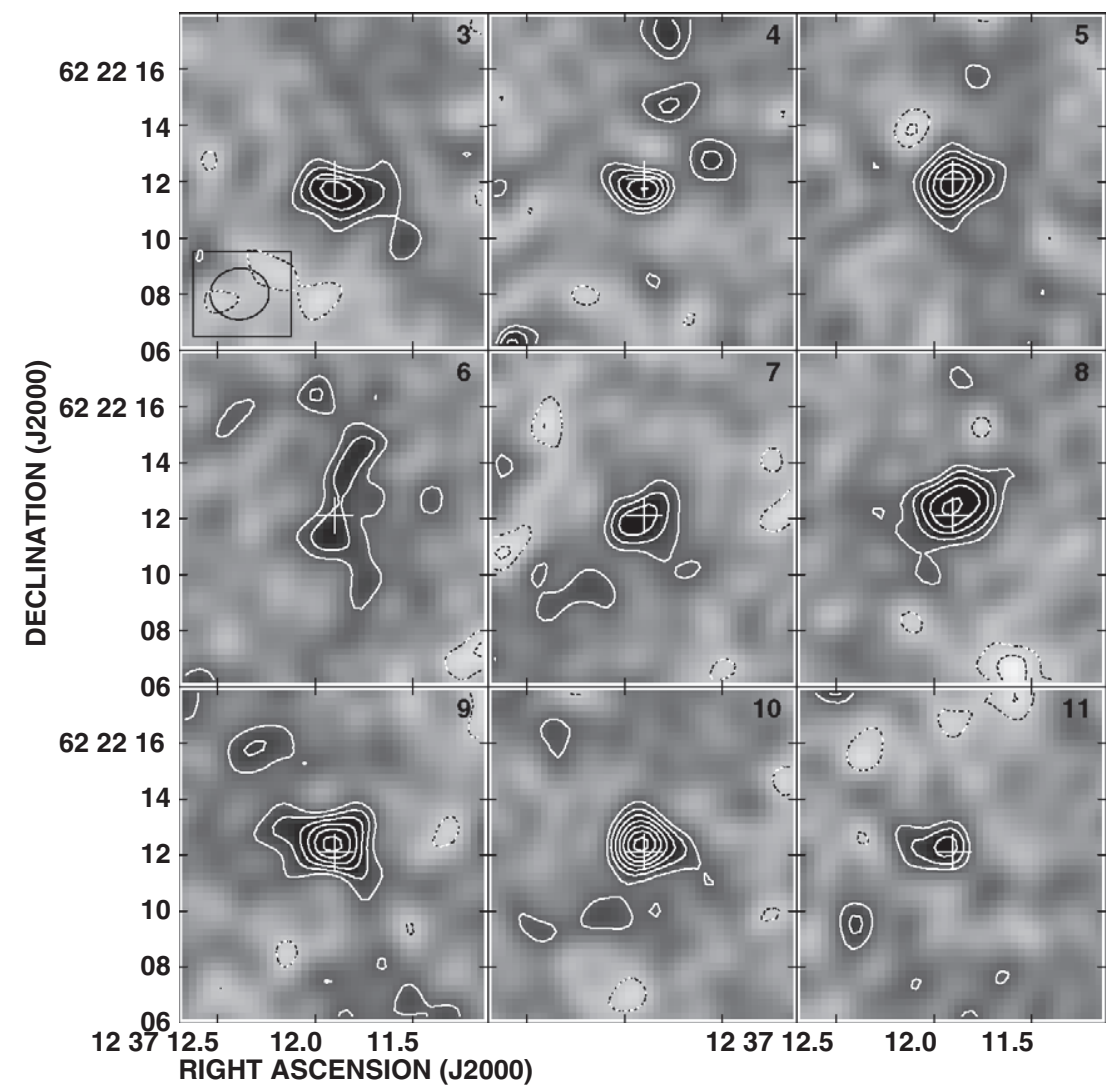

Figure 3. Channel images of the GN20 CO $2-1$ spectrum at $1^{\prime \prime} .9$ resolution and $78 \mathrm{~km} \mathrm{~s}^{-1}$ channel $^{-1}$. The contour levels are linear, in steps of $1 \sigma\left(0.12 \mathrm{mJy} \mathrm{beam}^{-1}\right)$, starting at $\pm 2 \sigma$. The channel labeled 3 is at $45.562 \mathrm{MHz}$, and each channel is $12 \mathrm{MHz}$ wide. Negative contours are dashed. 

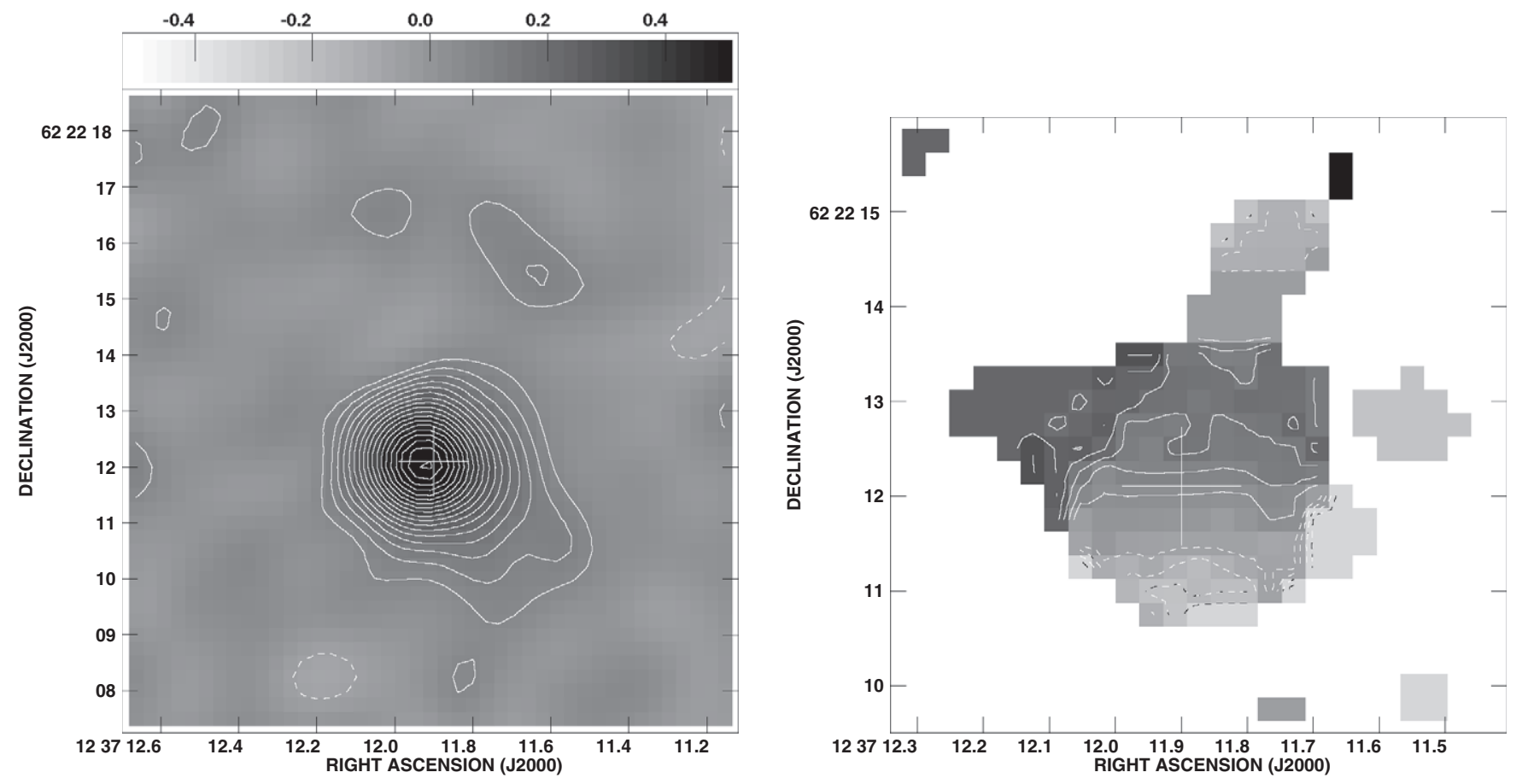

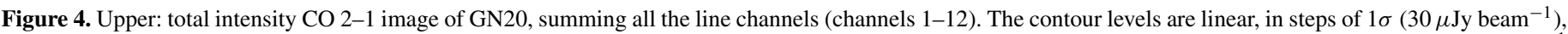

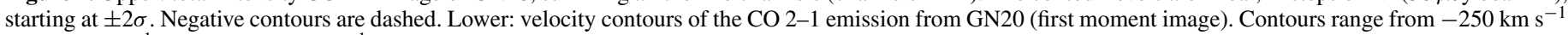
to $+250 \mathrm{~km} \mathrm{~s}^{-1}$, in steps of $50 \mathrm{~km} \mathrm{~s}^{-1}$, with zero velocity corresponding to $z=4.0556$. Negative velocities are dashed.

There are 11 other LBGs within our field, and with photometric redshifts that are consistent with the sampled range, although the $z_{\text {phot }}$ error bars are such that the galaxies could be outside the velocity range of the $\mathrm{CO}$ observations. We have looked for $\mathrm{CO}$ emission at all these positions, and find no significant detections to similar limits to those quoted above.

We emphasize that there is a large uncertainty in the derived $\mathrm{H}_{2}$ mass for the LBGs, due to the unknown conversion factor, $\alpha$. It is possible that metal-poor star-forming galaxies have a much larger value of $\alpha$ than ULIRGs or even the Milky Way due to $\mathrm{CO}$ dissociation by the more pervasive UV radiation field (Madden et al. 1997; Papadopoulos \& Pelupessy 2010).

\section{DISCUSSION}

We have detected $\mathrm{CO} 2-1$ emission from three galaxies in the GN20 proto-cluster at $z=4.05$ using the EVLA. The molecular gas masses range from $1.9 \times 10^{10} \times(\alpha / 0.8) M_{\odot}$ to $1.3 \times 10^{11} \times(\alpha / 0.8) M_{\odot}$. Hence, we are observing a group of molecular gas-rich galaxies undergoing extreme starbursts within $1.6 \mathrm{Gyr}$ of the big bang.

GN20 presents a particularly interesting case, given its very high luminosity and large spatial extent. We find that the sum of the stellar and gas mass in GN20 is comparable to the dynamical mass. While the assumptions involved in all three mass calculations are uncertain, it appears that the baryons likely dominate the mass content within $5 \mathrm{kpc}$ radius of GN20. Moreover, their is little room for a CO luminosity to gas mass conversion factor substantially larger than $0.8 M_{\odot}$ $\mathrm{K} \mathrm{km} \mathrm{s}{ }^{-1} \mathrm{pc}^{2}$. High-resolution observations are required to confirm the rotational aspect of the emission, and to obtain a more accurate derivation of the dynamical mass.

The morphology and velocity field for GN20 suggest a rotating disk, with a possible tidal tail extending $10 \mathrm{kpc}$ to the north in a single $78 \mathrm{~km} \mathrm{~s}^{-1}$ channel. We can speculate that GN20 is a gas-rich disk galaxy that has been gravitationally torqued by its neighboring galaxies. This gravitational disturbance has not disrupted the disk, but has greatly enhanced star formation in the disk, as well as generating the extended, tidal gas distribution.

Lastly, we have searched for CO emission from LBGs in the GN20 proto-cluster. We set an upper limit of $1.1 \times$ $10^{10} \times(\alpha / 0.8) M_{\odot}$ to the molecular gas mass in these galaxies, including one with an accurate spectroscopic redshift. Our limit is below the expected value based on the standard star formation law at this redshift, but within the broad scatter.

We are obtaining high-resolution $\mathrm{CO}$ imaging observations of the GN20 proto-cluster with the EVLA and the PdBI to perform a more detailed dynamical analysis, and study the spatial distribution of the $\mathrm{CO}$ excitation and gas-to-dust ratio of this forming cluster of massive galaxies at $z=4.05$.

E.D. acknowledges the funding support of ERC-StGUPGAL-240039 and ANR-08-JCJC-0008. D.R. acknowledges support from NASA through Hubble Fellowship grant HST-HF-51235.01 awarded by the Space Telescope Science Institute, which is operated by the Association of Universities for Research in Astronomy, Inc., for NASA, under contract NAS 5-26555.

\section{REFERENCES}

Aalto, S., Booth, R. S., Black, J. H., et al. 1995, A\&A, 300, 369 Andreon, S., \& Huertas-Company, M. 2011, A\&A, 526, 11 Aravena, M., Bertoldi, F., Carilli, C., et al. 2010, ApJ, 708, L36 Biggs, A., Younger, J., \& Ivison, R. 2010, MNRAS, 408, 342 Blain, A., Smail, I, Ivison, R., Kneib, J.-P., \& Frayer, D. 2002, Phys. Rep., 369, 111

Capak, P., Riechers, D., Scoville, N. Z., et al. 2011, Nature, 470, 233 Carilli, C. L., Daddi, E., Riechers, D., et al. 2010, ApJ, 714, 1407 Carilli, C. L., \& Holdaway, M. 1999, Radio Sci., 34, 817 Casey, C. M., Chapman, S. C., Daddi, E., et al. 2009, MNRAS, 400, 670 Chapman, S., Blain, A., Ibata, R., et al. 2009, ApJ, 691, 560 Chapman, S., Blain, A., Ivison, R., \& Smail, I. 2003, Nature, 422, 695 
Collins, D., Chris, A., Stott, John P., et al. 2009, Nature, 458, 603

Cornwell, T., Braun, R., \& Briggs, D. 1999, in ASP Conf. Ser. 180, Synthesis Imaging in Radio Astronomy II, ed. G. B. Taylor, C. L. Carilli, \& R. A Perley (San Francisco, CA: ASP), 151

Daddi, E., Dannerbauer, H., Krips, M., et al. 2009a, ApJ, 695, L176

Daddi, E., Dannerbauer, H., Stern, D., et al. 2009b, ApJ, 694, 1517

Daddi, E., Elbaz, D., Walter, F., et al. 2010, ApJ, 714, L118

Dannerbauer, H., Walter, F., \& Morrison, G. 2008, ApJ, 673, L127

Doherty, M., Tanaka, M., De Breuck, C., et al. 2009, A\&A, 509, 83

Downes, D., \& Solomon, P. M. 1998, ApJ, 507, 615

Hibbard, J., \& Mihos, C. 1995, AJ, 110, 140

Iono, D., Peck, A. B., Pope, A., et al. 2006, ApJ, 640, L1

Ivison, R., Papadopoulos, P., Smail, I., et al. 2011, MNRAS, 412, 1913

Ivison, R., Smail, I., Papadopoulos, P., et al. 2010, MNRAS, 404, 198

Kurk, J. 2009, A\&A, 504, 331

Madden, S., Poglitsch, A., Geis, N., et al. 1997, ApJ, 483, 200

Momjian, E., Carilli, C., \& Petric, A. 2005, AJ, 129, 1809

Momjian, E., Wang, W.-H., Knudsen, K. K., et al. 2010, AJ, 139, 1622
Moresco, M., Pozzetti, L., Cimatti, A., et al. 2010, A\&A, 524, 67

Morrison, G., Owen, F., Dickinson, M., Ivison, R., \& Ibar, E. 2010, ApJS, 188, 178

Papadopoulos, P., \& Pelupessy, F. 2010, ApJ, 717, 1037

Perley, R., Chandler, C., Butler, B., \& Wrobel, J. 2011, ApJ, 739, L1

Riechers, D. A., Hodge, J., Walter, F., Carilli, C. L., \& Bertoldi, F. 2011, ApJ, 739, L31

Renzini, A. 2006, ARA\&A, 44, 141

Riechers, D., Capak, P. L., Carilli, C. L., et al. 2010, ApJ, 720, L131

Shim, H., Chary, R.-R., Dickison, M., et al. 2011, ApJ, submitted (arXiv:1103.4124)

Solomon, P. M., \& Vanden Bout, P. A. 2005, ARA\&A, 43, 677

Stevens, J., Ivison, R. J., Dunlop, J. S., et al. 2003, Nature, 425, 264

Tacconi, L., Genzel, R., Smail, I., et al. 2008, ApJ, 680, 246

Tacconi, L., Neri, R., Chapman, S. C., et al. 2006, ApJ, 640, 228

Wardlow, J., et al. 2011, MNRAS, in press

Williams, C. C., Giavalisco, M., Porciani, C., et al. 2011, ApJ, 733, 92

Younger, J., Fazio, G. G., Wilner, D. J., et al. 2008, ApJ, 688, 59 